EESTI NSV TEADUSTE AKADEEMIA TOIMETISED 1955. IV kd., nr. 1 ИЗВЕСТИЯ АКАДЕМИИ НАУК ЭСТОНСКОИ ССР 1955. ТоМ IV, № 1

\title{
МЕТОД ВЫБОРА ЧИСЛА КОТЛОВ НА ТЭЦ И НА ОТОПИТЕЛЬНЫХ КОТЕЛЬНЫХ
}

\author{
Л. Э. ВАИК, \\ кандидат технических наук
}

Как известно, на городских теплоэлектроцентралях (ТЭЦ), а также на отопительных котельных, которые характеризуются в основном отопительно-вентиляционной тепловой нагрузкой, определение числа котлов производится с учетом возможного использования котла ремонтного резерва для покрытия пиковой части тепловой нагрузки. Такая возможность, однако, ограничена графиком планового текущего и капитального ремонта котлов.

До сих пор необходимое число котлов определялось по максимальной (расчетной) тепловой нагрузке котельной $\left(Q_{\text {кот }}^{\mathrm{p}}\right.$ мккал/час) и выбранной производительности единичного котла ( $D_{\kappa}$ т/час). Проверочным расчетом и построением графика планового ремонта котлов выяснялась правильность выбранного числа котлов. Таким образом, расчетным режимом для определения необходимого числа котлов являлась максимальная тепловая нагрузка котельной.

Такая методика слишком громоздка, главным образом из-за недостаточного выявления и учета влияния графика планового ремонта котлов на выбор числа котлов. Для облегчения соответствующих расчетов предлагается новый метод выбора числа котлов на ТЭЦ и на отопительных котельных $\left({ }^{2}\right)$, исходящий из попытки в аналитической форме учесть влияние графика планового ремонта котлов. Доказывается, что при учете графика планового ремонта в большинстве случаев расчетным режимом для определения числа котлов является тепловая нагрузка котельной $\left(Q_{\text {кот }}^{\prime}\right.$ мккал/час), при которой, в соответствии с графиком планового ремонта, необходимо один котел выключить из работы, потому что именно этот режим работы котельной до определенного числа котлов в котельной является наиболее напряженным.

Исходными положениями для определения необходимого числа котлов на ТЭЦ и отопительных котельных по предлагаемому методу являются:

1. На котельной не предусматривается установка специального котла ремонтного резерва.

2. На котельной устанавливаются только однотипные котлы равной производительности.

3. Продолжительность работы котельной во время пика теплового графика, когда тепловая нагрузка ее больше максимально-возможной 
тепловой нагрузки котельной при нахождении одного котла в текущем ремонте, не должна превышать максимально-возможную продолжительность одновременной непрерывной работы всех котлов.

По этим исходным положениям максимально-возможная продолжительность одновременной непрерывной работы всех котлов составляет

$$
h_{\text {макс }}=h_{\text {макс }}^{\prime}-h_{\mathrm{p}} n_{\kappa} \text { часов, }
$$

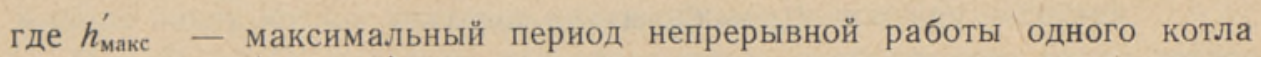
(в часах),

$h_{\mathrm{p}}$ - продолжительность текущего ремонта одного котла (в чаcax),

$n_{\mathrm{k}}$ - число котлов в котельной.

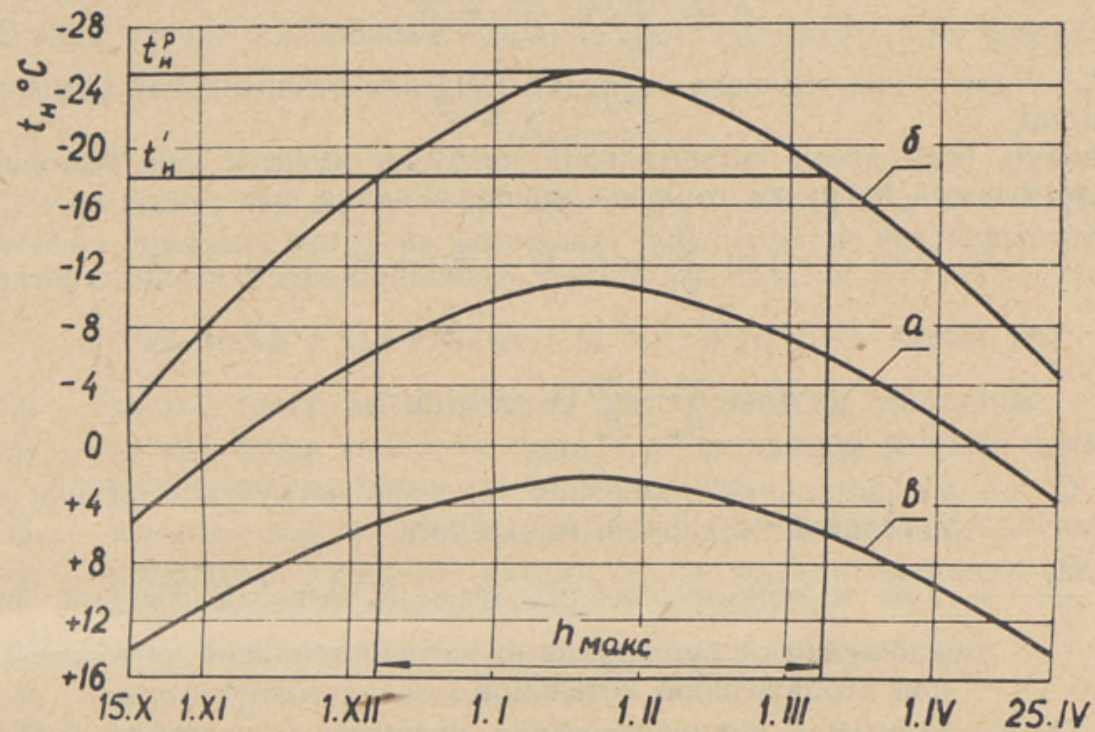

Фиг. 1. Изменение среднесуточных температур наружного воздуха за отопительный сезон. $a-$ среднее значение среднесуточных температур, 6 практическая граница минимальных значений среднесуточных температур, B - практическая граница максимальных значений среднесуточных температур.

Максимальная длительность периода одновременной непрерывной работы всех котлов $h_{\text {макс }}$ определяет на кривой среднесуточных температур наружного воздуха некоторую температуру наружного воздуха $t_{\mathrm{r}}^{\prime}$ (фиг. 1). Построение среднесуточных температур наружного воздуха по месяцам производится при этом по методу М. А. Басова $\left({ }^{1}\right)$.

По наружной температуре $t_{\mathrm{H}}^{\prime}$ можно определить соответствующую отопительную тепловую нагрузку в долях от расчетной отопительной тепловой нагрузки ТЭЦ или отопительной котельной по формуле

$$
a_{\mathrm{o \tau}}^{\prime}=\frac{Q_{\mathrm{o \tau}}^{\prime}}{Q_{\text {от }}}=\frac{t_{\mathrm{BH}}-t_{\mathrm{H}}^{\prime}}{t_{\mathrm{BH}}-t_{\mathrm{H}}^{\mathrm{p}}},
$$

где $Q_{\text {от }}^{\prime}$ - максимально-возможная отопительно-вентиляционная нагрузка ТЭЦ или отопительной котельной при нахождении одного котла в текущем ремонте, мккал/час, 


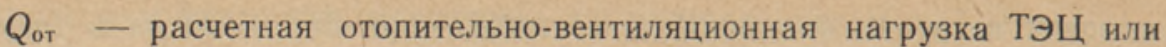
отопительной котельной, мккал/час,

$t_{\text {вн }}$ - внутренняя темнература в помещениях, ${ }^{\circ} \mathrm{C}$,

$t_{\mathrm{H}}^{\mathrm{p}}$ - расчетная температура наружного воздуха, ${ }^{\circ} \mathrm{C}$.

При чисто отопительно-вентиляционной нагрузке

$$
Q_{\text {от }}=Q_{\mathrm{p}} \text { мккал/час, }
$$

и доля тепловой нагрузки ТЭЦ или отопительной котельной при температуре наружного воздуха $t_{\mathrm{H}}^{\prime}\left(Q_{\mathrm{o \tau}}^{\prime}\right)$ от расчетной тепловой нагрузки $\left(Q_{\mathrm{p}}\right)$ выразится формулой

$$
a^{\prime}=\frac{Q_{\mathrm{oT}}^{\prime}}{Q_{\mathrm{p}}}=\frac{Q_{\mathrm{GT}}^{\prime}}{Q_{\mathrm{o \tau}}}=a_{\mathrm{o \tau}}^{\prime},
$$

где $Q_{\mathrm{p}}$ - расчетная тепловая нагрузка ТЭЦ или отопительной котельной, мккал/час.

Однако, если кроме отопительной нагрузки имеется еще бытовая и технологическая нагрузка горячего водоснабжения, то

$$
a^{\prime}=\frac{Q^{\prime}}{Q_{\mathrm{p}}}=a_{\text {oт }}^{\prime}\left(1-k_{6}\right)+k_{6}
$$

или

$$
a^{\prime}=\frac{t_{\mathrm{BH}}-t_{\mathrm{H}}^{\prime}}{t_{\mathrm{BH}}-t_{\mathrm{H}}^{\mathrm{p}}}\left(1-k_{6}\right)+k_{6},
$$

где $Q^{\prime}$ - максимальная ремонтная тепловая нагрузка ТЭЦ или отопительной котельной, мккал/час,

$k_{6}=\frac{Q_{6}}{Q_{\mathrm{p}}}-$ доля технологической нагрузки и нагрузки горячего водоснабжения от суммарной расчетной тепловой нагрузки ТЭЦ или отопительной котельной,

$Q_{6}$ - расчетная технологическая нагрузка и нагрузка горячего водоснабжения, мккал/час.

Поскольку $a^{\prime}$ выражает максимально-возможную тепловую нагрузку ТЭЦ или отопительной котельной в период ремонта котлов в долях от расчетной тепловой нагрузки, назовем в дальнейшем величину $\alpha^{\prime}$ коэффициентом максимальной ремонтной нагрузки.

При учете графика планового ремонта расчетный режим для выбора числа котлов определяется соотношением величин единичной производительности котлов $D_{\text {к }}$ т/час и «граничной» производительности котлов $D_{\kappa}^{\prime}$ т/час, определяемой по формуле

$$
\begin{gathered}
D_{\kappa}^{\prime}=\frac{10^{3}}{i_{1}-t_{\mathrm{n}}}\left(Q_{\text {кот }}^{\mathrm{p}}-Q_{\text {кот }}^{\prime}\right)=\frac{10^{3}}{i_{1}-t_{\mathrm{n}}}\left(Q_{\mathrm{p}}-Q^{\prime}\right)= \\
=\frac{10^{3}}{i_{1}-t_{\mathrm{n}}}\left(1-a^{\prime}\right) Q_{\mathrm{p}} \mathrm{T} / \text { час }
\end{gathered}
$$

где $i_{1}-$ теплосодержание свежего пара из котлов, ккал/кг,

$t_{\mathrm{n}}$ - температура питательной воды котлов, ${ }^{\circ} \mathrm{C}$,

$Q_{\text {кот }}^{\mathrm{p}}-$ расчетная тепловая нагрузка котельной, мккал/час,

$Q_{\text {кот }}^{\prime}$ - максимально-возможная тепловая нагрузка котельной в период ремонта котлов, мккал/час. 
Если единичная производительность котлов меньше или равна разности нагрузок котельной в паре при максимальном и ремонтном режимах, то есть при $D_{\kappa} \leqslant D_{\kappa}^{\prime}$, то расчетным режимом для выбора числа котлов является максимальная тепловая нагрузка ТЭЦ или отопительной котельной.

В противном случае, то есть при $D_{\kappa}>D_{\kappa}^{\prime}$, расчетным режимом для выбора числа котлов является максимальная ремонтная нагрузка.

При $D_{\kappa} \leqslant D_{\kappa}^{\prime}$ условием для определения необходимого числа котлов в котельной является

$$
n_{\mathrm{k}} D_{\mathrm{\kappa}}=D_{\text {кот }}^{\mathrm{p}} \mathrm{T} / \mathrm{чac},
$$

где $D_{\text {кот }}^{\mathrm{p}}-$ максимальная (расчетная) нагрузка котельной в паре, т/час.

В этом случае необходимое число котлов определяется по формулө

$$
n_{\mathrm{k}}=\frac{10^{3} \cdot Q_{\mathrm{KOT}}^{\mathrm{p}}}{D_{\mathrm{k}}\left(i_{1}-t_{\mathrm{n}}\right)} \text {. }
$$

Для отопительных котельных $Q_{\text {кот }}^{\mathrm{p}}=Q_{\mathrm{p}}$ мккал/час, а для ТЭЦ максимальная тепловая нагрузка котельной определяется по энергетической характеристике теплофикационных турбин (типа АТ и ВТ) $\left({ }^{3}\right)$ :

$$
Q_{\text {кот }}^{\mathrm{p}}=Q_{\ni}+Q_{\mathrm{p}}=n_{\mathrm{T}}\left(Q_{\mathrm{x}}+q_{\mathrm{K}} N_{\ni}-\beta Q_{\mathrm{T}}\right)+Q_{\mathrm{p}} \text { мккал/час, }
$$

где $Q_{\ni}$ - расход тепла на выработку электроэнергии, мккал/час,

$Q_{\mathrm{x}}$ - расход тепла холостого хода при смешанном режиме суммарно на электроэнергию и тепло, мккал/час,

$Q_{\tau}$ - номинальный расход тепла из отбора турбины, мккал/час,

$q$ к - частичный удельный расход тепла конденсационного цикла с включенным регулированием, мккал/мвт-ч,

$N_{\ni}$ - номинальная электрическая мощность турбины, мвт,

$n_{\mathrm{r}}$ - число турбоагрегатов на ТЭЦ,

$\boldsymbol{\beta}$ - коэффициент отбора, определяющий экономию тепла от комбинированной выработки электроэнергии и тепла по сравнению с раздельной работой этой же турбины по конденсационному циклу и отпуска тепла из котлов.

Наибольшее значение расчетной тепловой нагрузки ТЭЦ при данном числе и данной единичной производительности котлов определяется формулой

$$
Q_{\mathrm{p}-\mathrm{M}}=10^{-3} n_{\mathrm{\kappa}} D_{\mathrm{\kappa}}\left(i_{1}-t_{\mathrm{n}}\right)-n_{\mathrm{T}}\left(Q_{\mathrm{x}}+q_{\mathrm{\kappa}} N_{\mathrm{\Im}}-\beta Q_{\mathrm{\tau}}\right) \text { мккал/час. }
$$

«Граничной» производительности котлов соответствует определенное «граничное» число котлов в котельной, также определяющее расчетный режим для правильного выбора числа котлов; такое «граничное» число котлов

$$
n_{\mathrm{K}}^{\prime}=\frac{D_{\mathrm{koT}}^{\mathrm{p}}}{D_{\mathrm{K}}^{\prime}}=\frac{10^{3} \cdot Q_{\mathrm{KoT}}^{\mathrm{p}}}{D_{\mathrm{K}}^{\prime}\left(i_{1}-t_{\mathrm{n}}\right)}
$$

Подставляя в формулу (11) выражение $Q_{\text {кот }}^{\mathrm{p}}$ из (9) и выражение $D_{\text {к }}^{\prime}$ из (6), получаем после преобразований

$$
n_{\mathrm{\kappa}}^{\prime}=\frac{1}{1-a^{\prime}}\left(\frac{Q_{\ni}}{Q_{\mathrm{T}}} a_{\mathrm{T}}+1\right) .
$$


Численные значения величин, входящих в формулу (12), находятся обычно в следующих пределах: коэффициент максимальной ремонтной нагрузки $\alpha^{\prime}$ между 0,8 и 0,9 , коэффициент теплофикации $\alpha_{\text {т }}$ между 0,5 и 0,9 , а значение $\frac{Q_{\ni}}{Q_{\mathrm{T}}}$ для теплофикационных турбин типа АТ и ВТ в пределах $0,40-0,55$. При этих численных значениях «граничное» число котлов $n_{\mathrm{k}}^{\prime}$ находится в пределах 6-15.

Поскольку максимальная тепловая нагрузка ТЭЦ или отопительной котельной является расчетным режимом для выбора числа котлов только при условии

$$
n_{\kappa} \geqslant n_{\kappa}^{\prime}=6,7,8 \ldots 15,
$$

а число котлов на ТЭЦ и даже на отопительных котельных редко больше 6 , то из этого следует, что практически в большинстве случаев расчетным режимом для выбора числа котлов является не максимальная тепловая нагрузка $\left(Q_{\text {кот }}^{\mathrm{p}}\right)$, а максимальная ремонтная нагрузка котельной $\left(Q_{\text {кот }}^{\prime}\right)$.

Таким образом, основному случаю соответствует неравенство $D_{\kappa}>D_{\mathrm{K}}^{\prime}$, так что условием для определения необходимого числа котлов в котельной является

$$
\left(n_{\mathrm{\kappa}}-1\right) D_{\mathrm{\kappa}}=D_{\text {кот }}^{\prime} \mathrm{T} / \text { час. }
$$

В этом случае необходимое число котлов определяется по формуле

$$
n_{\mathrm{K}}=\frac{10^{3} \cdot Q_{\mathrm{KOT}}^{\prime}}{D_{\mathrm{K}}\left(i_{1}-t_{\mathrm{n}}\right)}+1 .
$$

Для отопительных котельных $Q_{\text {кот }}=Q^{\prime}=\alpha^{\prime} Q_{\mathrm{p}}$ мккал/час.

Однако при расчете максимальной ремонтной нагрузки котельной ТЭЦ возможны два случая, в зависимости от соотношения величин коэффициента максимальной ремонтной нагрузки ТЭЦ $\alpha^{\prime}$ и коэффициента теплофикации $a_{\tau}$, который означает долю тепловой нагрузки турбин в расчетной тепловой нагрузке ТЭЦ:

$$
a_{\mathrm{T}}=\frac{n_{\mathrm{T}} Q_{\mathrm{T}}}{Q_{\mathrm{p}}} .
$$

1. Если $a_{\tau} \leqslant \alpha^{\prime}$, то при переходе от расчетной наружной температуры $t_{\mathrm{H}}^{\mathrm{p}}$ к температуре $t_{\mathrm{н}}^{\prime}$ необходимая производительность котельной уменьшается только за счет уменьшения тепловой нагрузки ТЭЦ, а электроэнергия вырабатывается попрежнему полностью на базе тепловой нагрузки ТЭЦ.

2. Если $a_{\mathrm{\tau}}>a^{\prime}$, то при переходе от расчетной наружной температуры $t_{\mathrm{H}}^{\mathrm{p}}$ к температуре $t_{\mathrm{H}}^{\prime}$ необходимая производительность котельной уменьшается меньше, чем в первом случае, потому что с уменьшением тепловой нагрузки ТЭЦ в соответствии с повышением температуры наружного воздуха часть электроэнергии придется вырабатывать по конденсационному циклу (отборы турбин недогружены - экономия тепла от комбинированной выработки электроэнергии и тепла уменьшается).

Это иллюстрируется графиком тепловой нагрузки ТЭЦ $(Q)$ и котельной ТЭЦ $\left(Q_{\text {кот }}\right)$ по продолжительности при значении коэф- 
фициента максимальной ремонтной нагрузки $\alpha^{\prime}=0,75$ (фиг. 2). Так, например, при значении коэффициента теплофикации $a_{\mathrm{\tau}}=$ $=0,6<\alpha^{\prime}$ уменьшение тепловой нагрузки котельной ТЭЦ при переходе - от расчетной наружной температуры $t_{\mathrm{r}}^{\mathrm{p}} \mathrm{\kappa}$ температуре $t_{\mathrm{u}}^{\prime}$ определяется отрезком $Q_{\text {кот(",6) }}^{\text {p }} Q_{\text {кот }(0,6)}^{\prime}$, который равен отрезку $Q_{\mathrm{p}} Q^{\prime}$. При значении же коэффициента теплофикации $a_{\mathrm{\tau}}=1,0>\alpha^{\prime}$ уменьшение тепловой нагрузки котельной ТЭЦ при переходе от $t_{\mathrm{r}}^{\mathrm{p}}$ к $t_{\mathrm{H}}^{\prime}$ меньше, чем уменьшение тепловой нагрузки ТЭЦ, что видно на фиг. 2, где отрезок $Q_{\text {кот }(1,0)}^{\mathrm{p}} Q_{\text {кот }(1,0)}^{\prime}$ меньше отрезка $Q_{\mathrm{p}} Q^{\prime}$.

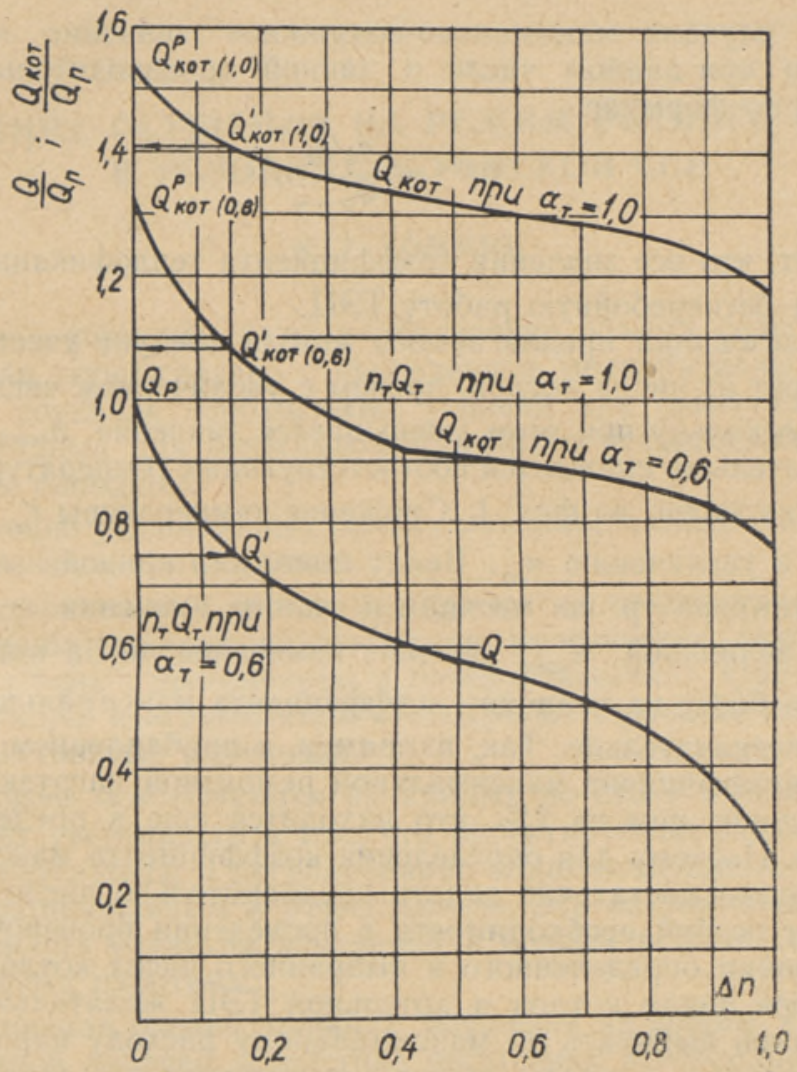

Фиг. 2. Тепловая нагрузка ТЭЦ $(Q)$ и котельной

ТЭЦ $\left(Q_{\text {кот }}\right)$ по продолжительности при значениях коэффициента теплофнкацин $\alpha_{\mathrm{T}} 0,6$ и 1,0 .

При $a_{\tau} \leqslant a^{\prime}$ максимальная ремонтная нагрузка котельной ТЭЦ выражается формулой

$$
Q_{\text {кот }}^{\prime}=n_{\mathrm{\tau}}\left(Q_{\mathrm{x}}+q_{\mathrm{\kappa}} N_{\ni}-\beta Q_{\mathrm{\tau}}\right)+a^{\prime} Q_{\mathrm{p}} \text { мккал/час. }
$$

Из (15) и (17) определяем наибольшее возможное значение расчетной тепловой нагрузки ТЭЦ при данном числе и данной пронзводительности котлов, обеспечивающее еще бесперебойную работу котельной:

$$
\begin{aligned}
Q_{\mathrm{p}-\mathrm{M}} & =\left[10^{-3}\left(n_{\mathrm{\kappa}}-1\right)\left(i_{1}-t_{\mathrm{n}}\right) D_{\mathrm{\kappa}}-n_{\mathrm{T}}\left(Q_{\mathrm{x}}+\right.\right. \\
& \left.\left.+q_{\mathrm{\kappa}} N_{\ni}-\beta Q_{\mathrm{T}}\right)\right] \frac{1}{\alpha^{\prime}} \text { мккал/час. }
\end{aligned}
$$


При $a_{\mathrm{\tau}}>a^{\prime}$ максимальная ремонтная нагрузка котельной ТЭЦ выражается формулой

$$
Q_{\text {кот }}^{\prime}=n_{\mathrm{\tau}}\left(Q_{\mathrm{x}}+q_{\mathrm{k}} N_{\mathrm{g}}\right)+a^{\prime} Q_{\mathrm{p}}(1-\beta) \text { мккал/час. }
$$

Наибольшее значение расчетной тепловой нагрузки ТЭЦ в этом случае при данном числе и данной производительности котлов определяется из (15) и (19):

$$
\begin{aligned}
Q_{\mathrm{p}-\mathrm{м}} & =\left[10^{-3}\left(n_{\mathrm{\kappa}}-1\right)\left(i_{1}-t_{\mathrm{n}}\right) D_{\mathrm{\kappa}}-n_{\mathrm{\tau}}\left(Q_{\mathrm{x}}+\right.\right. \\
& \left.\left.+q_{\mathrm{\kappa}} N_{\ni}\right)\right] \frac{1}{(1-\beta) \alpha^{\prime}} \text { мккал/час. }
\end{aligned}
$$

При всех случаях минимально-возможное значение коэффициента теплофикации при данном числе и данной производительности котлов определяется по формуле

$$
a_{\mathrm{T}}^{\text {мин }}=\frac{n_{\mathrm{T}} Q_{\mathrm{T}}}{Q_{\mathrm{p}-\mathrm{M}}},
$$

Это значит, что все значения коэффициента теплофикации $a_{\mathrm{\tau}} \geqslant a_{\tau}^{\text {мин }}$ обеспечивают бесперебойную работу ТЭЦ.

При использовании предлагаемого метода следует учесть, что значение $a_{\text {от }}^{\prime}$ зависит от числа котлов, причем с увеличением числа котлов оно

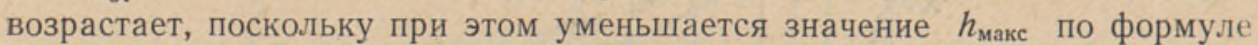
(1) и, следовательно, снижается соответствующая температура наружного воздуха $t_{\mathrm{H}}^{\prime}$, как видно из фиг. 1. Снижение температуры $t_{\mathrm{r}}^{\prime}$ приводит по формуле (2) к увеличению $a_{\text {ог }}^{\prime}$. Ввиду пологости кривой изменения среднесуточных температур по месяцам и малого значения величины $h_{\mathrm{p}}$ по сравнению с величиной $h_{\text {макс }}^{\prime}$ влияние изменения числа котлов на величину $t_{\mathrm{H}}^{\prime}$, и тем более на величину коэффициента максимальной ремонтной нагрузки $\alpha^{\prime}$, незначительно. Так, например, с прибавлением одного котла в котельной коэффициент максимальной ремонтной нагрузки $\alpha^{\prime}$ увеличивается не больше, чем на $2 \%$, что находится еще в пределах точности всего расчета. Поэтому для определения коэффициента максимальной ремонтной нагрузки достаточно задать предварительно число котлов в котельной и нет особой необходимости в проведении проверочного расчета при несовпадении определенного и выбранного чисел котлов.

При выборе числа котлов в котельной ТЭЦ желательно производительность котлов выбирать по максимальному расходу пара на теплофикационную турбину, а число котлов предварительно задать на единицу больше, чем число турбоагрегатов на ТЭЦ, то есть $n_{\text {предв }}=n_{\mathrm{r}}+1$.

Ннститут энергетики

Академии наук Эстонской ССР
Поступила в редакцию 29 XII 1953

\section{ЛИТЕРАТУРА}

1. М. А. Ба сов, Анализ повторяемости отопительной нагрузки для расчета теплогенерирующих установок, ДАН СССР, т. LXXII, № 4, 1950.

2. Л. Э. В а й к, Комплексные схемы энергоснабжения небольших городов, Диссертация, 1952.

3. Л. А. Мелен тьев, Теплофнкация, ч. I и ІІ, Изд. АН СССР, 1944, 1948. 